\title{
Status of detector development for the European XFEL
}

\author{
Jolanta Sztuk-Dambietz ${ }^{a}$, Steffen Hauf ${ }^{a}$, Andreas $\operatorname{Koch}^{a}$, Markus Kuster ${ }^{a}$, Monica Turcato ${ }^{a}$ \\ ${ }^{a}$ The European XFEL GmbH, Albert-Einstein-Ring 19, Hamburg, Germany;
}

\begin{abstract}
The European X-ray Free Electron Laser (XFEL.EU) will provide as-yet-unrivaled peak brilliance and ultrashort pulses of spatially coherent X-rays with a pulse length of less than $100 \mathrm{fs}$ in the energy range between 0.25 and $25 \mathrm{keV}$. The high radiation intensity and ultra-short pulse duration will open a window for novel scientific techniques and will allow to explore new phenomena in biology, chemistry, material science, as well as matter at high energy density, atomic, ion and molecular physics. The variety of scientific applications and especially the unique XFEL.EU time structure require adequate instrumentation to be developed in order to exploit the full potential of the light source. To make optimal use of the unprecedented capabilities of the European XFEL and master these vast technological challenges, the European XFEL GmbH has started a detector R\&D program. The technology concepts of the detector system presently under development are complementary in their performance and will cover the requirements of a large fraction of the scientific applications envisaged for the XFEL.EU facility. The actual status of the detector development projects which includes ultra-fast 2D imaging detectors, low repetition rate 2D detectors as well as strip detectors for e.g. spectroscopy applications and the infrastructure for the detectors' calibration and tests will be presented. Furthermore, an overview of the forthcoming implementation phase of the European XFEL in terms of detector R\&D will be given.
\end{abstract}

Keywords: XFEL.EU, X-ray detectors, FEL, X-rays, Free Electron Laser

\section{INTRODUCTION}

The European X-ray Free-Electron Laser (XFEL.EU) is a 3.4-kilometer-long international research facility currently under construction in the Hamburg, Germany area, that will start user operation in 2016!12 It is an X-ray photon source providing laterally coherent X-rays for six experimental stations (start-up configuration) in the range of approximately $250 \mathrm{eV}$ to $25 \mathrm{keV}$. An electron beam is accelerated on a linear path up to energies of $17.5 \mathrm{GeV}$ by superconducting cavities. The electrons then generate coherent X-rays in a series of undulators up to $200 \mathrm{~m}$ in length based on the SASE process (Self-Amplified Spontaneous Emission). The electron acceleration, photon beam generation and beam transport path extends over a total length of $3.4 \mathrm{~km}$ providing particularly intense and short X-ray pulses down to a few femtoseconds with a peak brilliance of $10^{33}$ photons $/ \mathrm{s} / \mathrm{mm}^{2} / \mathrm{mrad}^{2} / 0.1 \% \mathrm{BW}$. The electron source will deliver trains of typically 2700 pulses at a pulse repetition rate of 4.5 MHz. Each train will be followed by a gap of $99.4 \mathrm{~ms}$. The unique features of the European XFEL drive the need to develop dedicated instrumentation and detectors in particular. The instruments are optimized for particular purposes. Each experiment requires light sources with special properties, such that the instruments are permanently assigned to the different X-ray sources (beamlines) of the European XFEL. There are six scientific instruments planned to be installed in the start-up phase of the project. The list of the start-up instruments is shown in the Table 1

\section{DETECTORS AT THE XFEL.EU SCIENTIFIC INSTRUMENTS}

The different scientific applications at the XFEL.EU will require different instrumentation, in particular different imaging (2D) and spectroscopy (1D) detectors. Tables 2 and 3 show the summary of the main detector requirements for all scientific instruments which are foreseen at the XFEL.EU.

Most challenging are high-repetition rate 2D imaging detectors. Therefore, concerning the high frame rate and associated data rate, three detector programs are presently working towards producing $2 \mathrm{D}$ area detectors

Further author information: (Send correspondence to J. Sztuk-Dambietz)

J. Sztuk-Dambietz.: E-mail: jolanta.sztuk@xfel.eu, Telephone: +49 4089986925 
Table 1. Scientific instruments at the XFEL.EU.

\begin{tabular}{|l|l|}
\hline Instrument & Main application \\
\hline \multicolumn{2}{|c|}{ SASE 1 $E \in 3-20 \mathrm{keV}$} \\
\hline $\begin{array}{l}\text { Single Particles, clusters, } \\
\text { and Biomolecules (SPB) }{ }^{3}\end{array}$ & $\begin{array}{l}\text { Structure determination of single particles - atomic clusters, } \\
\text { biomolecules, virus particles, cells. }\end{array}$ \\
\hline $\begin{array}{l}\text { Femtosecond X-ray Experi- } \\
\text { ments (FXE) }\end{array}$ & $\begin{array}{l}\text { Time-resolved structure investigations and dynamics investi- } \\
\text { gation of solids, liquids, gases. }\end{array}$ \\
\hline \multicolumn{2}{|c|}{ SASE 2 $E \in 3-24(36) \mathrm{keV}$} \\
\hline $\begin{array}{l}\text { Materials Imaging and Dy- } \\
\text { namics (MID) }\end{array}$ & $\begin{array}{l}\text { Structure determination of nano-devices and dynamics at } \\
\text { nano-scale }\end{array}$ \\
\hline $\begin{array}{l}\text { High-Energy Density mat- } \\
\text { ter experiments (HED) } 6\end{array}$ & $\begin{array}{l}\text { Investigation and excitations of matter under extreme condi- } \\
\text { tions using hard X-ray FEL radiation, e.g. for probing dense } \\
\text { plasma. }\end{array}$ \\
\hline $\begin{array}{l}\text { Small Quantum Systems } \\
\text { (SQS) }\end{array}$ & $\begin{array}{l}\text { Ultra-fast investigation (spectroscopy) of atoms, ions, } \\
\text { molecules and clusters in intense fields and non-linear phe- } \\
\text { nomena. }\end{array}$ \\
\hline $\begin{array}{l}\text { Spectroscopy \& Coherent } \\
\text { Scattering (SCS) } 8\end{array}$ & $\begin{array}{l}\text { Structure and dynamics of nano-systems and of non- } \\
\text { reproducible biological objects. Electronic and atomic struc- } \\
\text { ture as well as dynamics of soft and hard matter, bio species } \\
\text { and nanomaterials using soft X-rays. }\end{array}$ \\
\hline
\end{tabular}

for the European XFEL. Out of these three programs, two will provide detectors optimized for the hard X-ray regime, with optimal performance at photon energies above $10 \mathrm{keV}$ : the Large Pixel Detector (LPD) and the Adaptive Gain Integrating Detector (AGIPD). The DEPFET sensor with Signal Compression (DSSC) is designed for experiments using lower energy X-rays, down to a few hundred $\mathrm{eV}$.

The LPD detector,$\frac{9}{9}$ to be used at the FXE instrument, achieves the front-end required large dynamic range by using three different gain settings in parallel, each followed by its own analogue pipeline. In order to implement three analogue pipelines with 512 storage cells each in parallel per pixel, the pixel size has to be sufficiently larger, e.g. $500 \mu \mathrm{m} \times 500 \mu \mathrm{m}$. The LPD front-end module will include an interposer between the silicon sensor and the ASIC, which gives the flexibility to have different pixel sizes and layouts between the silicon sensor and the ASIC. Extra radiation shielding between the sensor and the part of the ASIC, e.g. the memory cells, relaxes the required radiation hardness of the ASIC. The LPD two-tile prototype system was delivered to the XFEL.EU in March 2013 and is under test there. Dedicated X-ray beam tests at the PETRA III synchrotron at DESY and at the linear accelerator LCLS of the prototype are planned for May 2013.

The AGIPD detector ${ }^{10} 11$ consists of a classical hybrid pixel array, with readout ASICs bump-bonded to a silicon sensor. The ASIC is designed in $130 \mathrm{~nm}$ CMOS technology and uses dynamic gain switching to cover the large dynamic range $\left(10^{4}\right.$ photons at $\left.E_{\gamma}=12 \mathrm{keV}\right)$, and an analogue memory to store recorded images during the $0.6 \mathrm{~ms}$ duration of the bunch train. The images are subsequently read out and digitized during the $99.4 \mathrm{~ms}$ interval between bunch trains. The analogue memory consists of two types of storage cells: for amplitude values and for the encoded gain settings. The analogue memory is designed to store 352 images, i.e. 352 samples per pixel of size $200 \mu \mathrm{m} \times 200 \mu \mathrm{m}$. To optimize the use of this limited storage depth by overwriting unfit/obsolete images, the memory is operated in random access mode. The detector components (sensor and ASIC) are optimized to be radiation tolerant 12, 13 To verify the performance of the detector, the latest chip assembly (AGIPD0.4) was tested at the P10 beameline of the PETRA III synchrotron at DESY 14 The required high dynamic range, $\left(10^{4}\right.$ photons), was demonstrated and the measured ENC noise was less than $340 \mathrm{e}^{-}$. The results are in agreement with the expected detector performance. The AGIPD detector is foreseen as the primary detector for the SPB and MID instruments.

The DSSC 15 uses a non-linear response of the active sensor pixels to cope with the large dynamic range, and 
Table 2. Imaging detector requirements for the scientific instruments at the XFEL.EU.

\begin{tabular}{|c|c|c|c|c|c|c|}
\hline & SPB & FXE & MID & HED & SQS & SCS \\
\hline Requirements & \multicolumn{6}{|c|}{ Large Area Imaging Detectors } \\
\hline Repetition rate & \multicolumn{6}{|c|}{ up to $4.5 \mathrm{MHz}$} \\
\hline $\begin{array}{l}\text { Sensitive energy } \\
\text { range (Quantum } \\
\text { Efficiency (QE) } \\
>80 \%\end{array}$ & $3-16 \mathrm{keV}$ & $6-25 \mathrm{keV}$ & $3-25 \mathrm{keV}$ & $4-25 \mathrm{keV}$ & $0.26-3 \mathrm{keV}$ & $0.26-3 \mathrm{keV}$ \\
\hline Pixel size & $\leq 200 \mu m$ & $\leq 500 \mu m$ & $\leq 50 \mu m$ & $\leq 100 \mu m$ & $\leq 200 \mu m$ & $\leq 200 \mu m$ \\
\hline Pixel number & \multicolumn{6}{|c|}{$1000 \times 1000$ or more } \\
\hline $\begin{array}{l}\text { Radiation hard } \\
\text { design }\end{array}$ & \multicolumn{6}{|c|}{ yes } \\
\hline $\begin{array}{l}\text { Single photon } \\
\text { sensitivity }\end{array}$ & \multicolumn{6}{|c|}{ yes } \\
\hline Dynamic range & $\geq 10^{6}$ & $10^{5}$ & $\geq 10^{3}$ & $\geq 10^{3}$ & $\geq 10^{3}$ & $\geq 10^{3}$ \\
\hline $\begin{array}{l}\text { Vacuum com- } \\
\text { patibility }\end{array}$ & $10^{-3} \mathrm{mbar}$ & $\begin{array}{l}\text { gas/ambient } \\
\text { pressure }\end{array}$ & $10^{-3} \mathrm{mbar}$ & \multicolumn{3}{|c|}{$10^{-6}$ mbar } \\
\hline $\begin{array}{l}\text { Variable central } \\
\text { hole }\end{array}$ & \multicolumn{6}{|c|}{ yes } \\
\hline $\begin{array}{l}\text { VETO capabil- } \\
\text { ity }\end{array}$ & \multicolumn{6}{|c|}{ yes } \\
\hline
\end{tabular}

a digital memory to store images inside the pixels. An advantage of the DEPFET is the low noise performance, which makes this detector well suited for experiments using lower energy X-rays, down to a few hundred eV. The DSSC design foresees hexagonal pixels (pixel pitch $204 \mu \mathrm{m} \times 236 \mu \mathrm{m}$ ), which give a more homogeneous drift field and a faster charge collection than square pixels. The DEPFET sensor will be bump bonded to mixed signal readout ASICs. The ASICs are designed in 130nm CMOS technology and provides fully parallel readout of the DEPFET pixels. The signals coming from the detector, after having been processed by an analogue filter, are immediately digitized and locally stored in a custom-designed memory also integrated in the ASICs. The advantage of digital storage over analogue is the absence of signal leakage. The number of stored frames per macro-bunch is expected to be 640. This detector is planned to be part of the SQS and SCS instruments. The first sensors with the final non-linear characteristic will be ready in spring, the full-scale chip submission is planned for this summer.

In parallel to the technology development of detectors, their integration into XFEL.EU in terms of mechanics is ongoing. Similarly, work on the calibration strategies and software development is progressing. A calibration group, which includes representatives from each of the consortia as well as the XFEL.EU Detector Development Group members has been set up. The goal of the group is to define the detector parameters to be calibrated and the required accuracy, to develop the strategies to calibrate the different types of detectors, define and build the infrastructure needed for calibration and characterization of the different types of detectors, to define calibration and test (re-calibration) procedures, to define and implement the calibration data format and to structure and develop and implement calibration software within the XFEL.EU software framework (Karabo). More details about the detector test infrastructure will be given in Section 4

In the following subsection we will focus on the detector geometry requirements driven by the two scientific instruments where the AGIPD detector will be used as a primary detector, SPB and MID. 
Table 3. Strip detector requirements for the XFEL.EU scientific instruments at SASE 1 and SASE 2 (hard-X-rays).

\begin{tabular}{|c|c|c|c|c|}
\hline & SPB & FXE & MID & HED \\
\hline Requirements & \multicolumn{4}{|c|}{ Strip detectors for hard X-rays } \\
\hline Repetition rate & \multicolumn{4}{|c|}{ up to $4.5 \mathrm{MHz}$} \\
\hline $\begin{array}{l}\text { Sensitive energy } \\
\text { range }\end{array}$ & $3-16 \mathrm{keV}$ & $6-25 \mathrm{keV}$ & $6-25 \mathrm{keV}$ & $4-25 \mathrm{keV}$ \\
\hline Strip pitch & \multicolumn{4}{|c|}{$50 \mu \mathrm{m}$} \\
\hline Strip number & \multicolumn{4}{|c|}{$\sim 1000$} \\
\hline $\begin{array}{l}\text { Radiation hard } \\
\text { design }\end{array}$ & \multicolumn{4}{|c|}{ no direct beam } \\
\hline $\begin{array}{l}\text { Single photon } \\
\text { sensitivity }\end{array}$ & \multicolumn{4}{|c|}{ yes } \\
\hline Dynamic range & \multicolumn{4}{|c|}{$10^{4}$} \\
\hline $\begin{array}{l}\text { Vacuum com- } \\
\text { patibility }\end{array}$ & \multicolumn{4}{|c|}{$10^{-5} \mathrm{mbar}$} \\
\hline $\begin{array}{l}\text { VETO capabil- } \\
\text { ity }\end{array}$ & \multicolumn{4}{|c|}{ yes } \\
\hline
\end{tabular}

\subsection{Detector geometry at the SPB instrument}

The main scientific cases currently foreseen at the SPB instrument are crystallography of biological nano-crystals (NX) as well as coherent diffractive imaging (CDI) of both reproducible and non-reproducible particles.

One of the key potentials of CDI lies in its ability to provide quantitative image contrast, so that each reconstructed real space pixel can be associated with its sample electron density. This imaging modality is possible only if certain requirements of the detection geometry are fulfilled. As a necessary condition, the diffraction pattern needs to be sampled on a fine enough grid to record its full information content. In addition, the high-intensity scattering contributions close to the center of the diffraction pattern need to be measured as completely as possible. Any missing information due to the passage of the central beam through the central detector hole, or due to other blind regions introduced by the modular design of the detectors (non-sensitive areas in between detector modules) can distort or prevent a quantitative reconstruction ${ }^{[16}$ Another demanding requirement in CDI, particularly for larger samples such as viruses or cells, is the high intensity range of diffraction patterns. ${ }^{[17}$ Detectors with a dynamic range of seven orders of magnitude or more are required to cover the full dynamic range of the diffraction pattern for strongly scattering samples. This requirement, however, exceeds the maximum dynamic range of even the newest integrating detectors suitable for XFEL.EU applications by a few orders of magnitude. It was shown ${ }^{18}$ for non-crystalline particles that both the mentioned problems, i.e. the missing data regions and the high intensity range of certain diffraction patterns, can be mitigated by utilizing two detector planes: one device close to the sample with a relatively large active area and central hole $(2.5 \mathrm{~cm})$, to measure the high-resolution diffraction data, and one smaller rear device at about twice or more the distance from the sample to measure the small-angle diffraction. This second device also needs a central gap or hole to allow for the passage of the direct beam, which is eventually blocked by a beam dump at the end of the experimental hutch. The two detectors can operate at different gain settings, by e.g. using filters so that the larger dynamic range which was brought up as a requirement is also addressed.

Another critical issue is that the resolution in crystallography is geometrically limited by the numerical aperture covered by the detector at a certain distance to the sample. If scattering from the sample exceeds the detector area, the resolution is said to be geometrically limited. In a crystal, in contrast to biological single particles, the signal is strongly enhanced by the large number of unit cells. The crystallographic resolution of an 
instrument is given by the following equation. 19

$$
\Delta x=\frac{\lambda}{2 \cdot \sin \left(\frac{2 \theta}{2}\right)},
$$

where $\lambda$ is the wavelength and $2 \theta$ is the diffraction angle with respect to the optical axis. Therefore, the resolution as a function of the detector-sample distance is:

$$
z=\frac{D}{2 \cdot \tan \left(2 \cdot \arcsin \left(\frac{\lambda}{2 \Delta x}\right)\right)},
$$

where $D$ is the distance between two opposing outermost pixels on the detector. Given the feasibility of $1.9 \AA$ crystallographic resolution for NX at LCLS, 20 a resolution of at least 1.5-2 $\AA$ should be provided at the XFEL.EU instrument. Taking into account the geometry of the AGIPD detector, minimum sample-detector distances of $z_{\text {min }}=13.3-19.4 \mathrm{~cm}$ at a photon energy of $12 \mathrm{keV}$, and smaller for lower energies are required! 19

\subsection{Angular resolution and pixel size at the MID instrument}

One of the most critical parameters for the science cases at the MID instrument is the angular resolution, which is determined by the pixel size and the sample-to-detector distance. X-ray Photon Correlation Spectroscopy (XPCS), a technique that studies the slow dynamics of various equilibrium and non-equilibrium processes occuring in condensed matter systems, is the method which requires the highest angular resolution of all applications. A detector with a pixel size of $200 \mu \mathrm{m} \times 200 \mu \mathrm{m}$, is not suitable for the scientific cases where a high angular resolution is required. In order to get the highest quality time-correlated data, not more than one speckle per pixel is needed. Therefore, the pixel size should be equal or smaller than the speckle size. A possible solution can be the use of commercial detectors with sufficient small pixel size but much lower readout rate than $4.5 \mathrm{MHz}$ which are also being developed to mitigate the risks for day-one operation e.g. the Fast CCD 21 and pnCCD!22 The Fast CCD has a limited dynamic range but a small pixel size $\left(30 \times 30 \mu \mathrm{m}^{2}\right)$. As the detector was designed for soft X-rays, particular attention has to be paid when running with hard X-ray photons in terms of radiation damage. In addition, it has to be considered that the quantum efficiency at hard X-ray energies is limited due to the sensor thickness of $200 \mu \mathrm{m} \mathrm{Si}$. The potential for further developing the detector for hard X-ray experiments is being investigated. Of particular interest would be the use of a thicker sensor increasing the efficiency and obtaining a larger dynamic range.

\section{OUTLOOK TO OTHER DETECTOR PROJECTS}

\subsection{Detectors for spectroscopy and diagnostics}

The unique features of the XFEL.EU, in particular the very high repetition rate, represent a challenge for the large-area imaging detectors but also for the smaller-area detectors and make the use of standard commercial devices impossible. Dedicated solutions are therefore envisaged for small imaging- or strip-detectors. At the moment, two particular 4.5 MHz small-area detector solutions are under study and are planned to be used at the XFEL.EU: a strip detector for hard X-rays and an imaging detector for soft X-rays. Hard X-rays photon-beam diagnostics as well as hard X-ray absorption and emission spectroscopy at the European XFEL make use of strip detectors as detectors for beam spectrometers or as energy-dispersive detectors in combination with a crystal as energy-dispersive element. The European XFEL is establishing cooperation with the Paul Scherrer Institute in Villigen to develop a new version of the Gotthard detector well suited for the XFEL.EU needs. Starting from the present detector version, the modifications planned to adapt it to XFEL.EU running conditions include the capability of running at an increased frame rate and to provide a veto signal in order to be able to remove non-interesting images. In another particular application, resonant inelastic X-ray scattering (RIXS), a MicroChannel Plate detector matched to a delay-line readout is foreseen to be used. In this case the European XFEL is planning to use a highly customized solution provided by a German Company. The science-driven detector specifications have been defined and the expected detector performance estimated. The detector will be able to record, at $4.5 \mathrm{MHz}$ repetition rate, the photons emitted by the investigated sample, expected to be not more than 250 towards the detector with a resolution of the order of $50 \mu \mathrm{m}$ in both directions. 


\subsection{Future developments}

Although the XFEL.EU will start user operation in 2016 and the three main 2D detector projects are expected to cover the need of the scientists for the first years of running, plans for the next generation of detectors are being made. The need for smaller pixel sizes, higher dynamic range and the possibility of running at $4.5 \mathrm{MHz}$ and to record all the 2700 pulses of a macro bunch are the main motivations that push the search for new technology solutions. The change from 130 to $65 \mathrm{~nm}$ CMOS technology and three-dimensional integrated electronic circuits can contribute to this challenge. The development of edgeless sensors can help in minimising the inactive detector area. Moreover, the need to have high quantum efficiency also at energies greater than $20 \mathrm{keV}$ motivate the use materials with higher atomic numbers than silicon for the detectors' active areas.

\section{DETECTOR TEST INFRASTRUCTURE}

Accurate calibration and characterization of the different detectors as well as development of user-friendly procedures and tools to re-do calibration during the XFEL.EU operation phase are very important for the success of the project. The detector group at XFEL.EU works on a dedicated test environment for fully assembled detectors, detector modules and/or sensor tiles which can be used during the start-up phase of the project and during the operation phase of the European XFEL facility. The test environment will allow to do functional tests as well as calibration and performance characterization of the detectors in use at the experimental stations of XFEL.EU. Since the detector systems foreseen for XFEL.EU consists of components sensitive to surface and particle contamination, a clean and controlled infrastructure will be provided for calibration, characterization and testing of detectors and detector components. Especially for detectors for low energy applications at photon energies below $2 \mathrm{keV}$ and for handling and storage of e.g. MCPs, a controlled and clean environment is essential. For the detector development program of the Detector Group at the XFEL.EU we aim for a calibration, integration, inspection and cleaning/wet chemistry laboratory in a clean room in the XFEL.EU headquarter building (XHQ) in Schenefeld.

As the main laboratory in Schenefeld will not be ready before 2015, the XFEL.EU detector group is temporarily using one of the DESY experimental halls. The work on developing the infrastructure needed for detector characterization is in progress.

\subsection{X-ray sources for detector calibration and characterization}

To achieve precise results, the characterization and the detailed calibration of the 2D X-ray cameras, strip and other X-ray sensitive detectors have to be performed using X-ray photons, with characteristics similar to the real conditions at the XFEL.EU experiments. The X-ray generators and sources, which will be used for calibration, have to fulfill the following requirements:

- The time structure of the X-rays has to be similar or as close as possible to that of the XFEL.EU. Ideally, short ( $\leq 50 \mathrm{~ns}$ ) X-ray pulses created with a repetition rate of up to $4.5 \mathrm{MHz}$ will be required. X-ray sources, which are capable to reproduce the XFEL.EU time structure (repetition rate, train structure) will be useful to test the timing properties and pulse-to-pulse response of the detection systems, e.g. the vetoing of a pre-defined pulse.

- The X-ray energy of the source shall be similar to that used at the XFEL.EU experiments, i.e. 0.5 to $25 \mathrm{keV}$. This is particularly important for detector performance parameters, which strongly depend on the $\mathrm{X}$-ray photon energy. Ideally the source should be monochromatic, i.e. with an energy spread significantly smaller than the energy resolution of the detector.

- The intensity of the X-ray beam, which can be defined as:

$$
I=\frac{N_{\gamma}}{A \cdot \tau}
$$

where $N_{\gamma}$ is number of photons, $A$ is the unit area (e.g. pixel size) and $\tau$ is the unit time (e.g. pulse length), shall be adjustable and vary from one photon pixel ${ }^{-1}$ pulse $^{-1}$ up to $10^{4}$ photons pixel $^{-1}$ pulse $^{-1}$ 
at the detector head to be able to calibrate and characterize the detector response over a wide dynamic range. Ideally, the X-ray intensity shall be stable in time to a level of $1 \% \mathrm{~h}^{-1}$ at photon intensities $I=10^{4}$ photons pixel $^{-1}$. In general, the intensity stability of the source has to be better than the Poisson noise $\left(\sqrt{\left(N_{\gamma}\right)}\right.$.

- Illumination of the detector surface:

- Point illumination: point illumination means that illumination of a single pixel/strip shall be possible. For the actual generation of imaging detectors under development for XFEL, we require an X-ray spot with a diameter of $\leq 20 \mu \mathrm{m}$.

- Cluster illumination: illumination of a cluster of pixels shall be possible to test pixel-to-pixel interaction (e.g. cross talk). Uniform illumination with X-rays with known emission characteristics on up to $16 \times 16$ pixels is sufficient.

- Uniform flat field illumination: to study the uniformity of the response of the area detectors uniform flat field illumination shall be possible with homogeneity of the order of $10 \%$ for low spatial frequency variations. Ideally the flat field size should be large enough to illuminate an entire active area of a detector or detector module, i.e. approximately $10 \mathrm{~cm} \mathrm{x} 10 \mathrm{~cm}$ or larger. Since this is technically difficult or impossible to realize, smaller flat field sizes together with the possibility to move the detector through the X-ray beam are an alternative option.

- The X-ray test setup shall allow testing and operating detector systems under different conditions:

- Vacuum operation: for testing of detectors sensitive to low energy photons, $\mathrm{E} \leq 1 \mathrm{keV}$, a clean environment is required to e.g. avoid surface contamination of the entrance window. Consequently the test setup shall be able to be operated at a pressure lower than $10^{-5}$ mbar. The time to vent and pump the test setup shall be a few hours at maximum to reduce overhead times to a minimum.

- Atmospheric pressure operation: for detectors being sensitive to photons with energy above 3 keV, it is sufficient to operate the detector in air or inert gas at ambient pressure.

It is very challenging to build an X-ray test environment that meets all the requirements summarized above. Therefore, we consider using different sources and test setups which are complementary to each other. Currently two types of sources are considered for laboratory calibration and characterization of the XFEL.EU detectors: radioactive isotopes and X-ray/electron generators. Long-lived radioactive isotopes are convenient X-ray sources, providing stable intensity (half-life of the radioactive isotope $\gg$ time needed for measurement) and characteristic emission lines with well-defined photon energies. In addition, they are compact, easy to operate and inexpensive in comparison to other X-ray generators. Radioactive sources can be used for flat-field and point illumination if used in combination with a collimator and pinhole mask. The achievable intensities are moderate and limited due to the activity of the available isotope sources. X-ray generators will be used whenever higher X-ray intensities are required and shall provide multiple-quanta energies. The commercially available diffraction or industrial X-ray tubes are mostly operated in continuous mode or at low repetition rates and thus are not sufficient for the characterization of the detector parameters which may depend on the repetition rate. Nevertheless they can be used to calibrate some of the detector parameters (e.g. gain conversion). We consider small portable X-ray generators like the Mini-X from AMPTEK Inc..

To be able to characterize the features of the detectors which are mostly connected with the European XFEL unique time structure and beam intensity we are planning to use FELs or suitable synchrotrons beams. For such tests where the high intensity of the source is not so important, we are considering to build a custom pulsed multitarget X-ray generator.

The main subcomponents of the such custom generator are the following:

- Electron source: the electron gun shall provide a pulsed electron beam with electron energies of up to a few tens of $\mathrm{keV}$, adjustable in intensity and spot size. We require a beam current adjustable from $1 \mathrm{nA}$ to $20 \mathrm{~mA}$ and a spot-size ranging from $0.1 \mathrm{~mm}$ (focused column, spot) to $10 \mathrm{~mm}$ (flood beams). The electron 
gun shall provide pulse widths from approximately $50 \mathrm{~ns}$ up to $0.1 \mathrm{~ms}$. Ideally we require two electron guns, which can be operated in parallel or successively providing two electron pulses with a distance equivalent to the XFEL.EU pulse-to-pulse distance of 220 ns.

- Multi-Target Anode for X-ray production: the anode should provide space for approximately ten different target materials. It is attached to the shaft of a motor rotating the anode to choose the required target material. During operation the anode should stay in a fixed position. Due to the high electron currents needed to generate high X-ray intensities, the anode wheel needs to be cooled. Most likely a liquid cooling system is required.

The X-ray test environment will provide an experimental setup to test and characterize detectors under vacuum and ambient conditions with a high intensity pulsed or continuous X-ray beam. The test environment will be designed in such a way that several different kinds of X-ray sources can be operated, e.g. radioactive isotopes or an X-ray generator. It will consist of several subcomponents i.e. a X-ray source, a short beam line of a length of 2 to $3 \mathrm{~m}$ and a test chamber for vacuum and ambient operation.

\subsubsection{Monte Carlo simulation for pulsed multi-target X-ray generator}

An accurate description of the X-ray spectra emitted from targets irradiated by kilo-electron-volt (keV) electron beams is of interest for the design and later for the optimization and characterization of the X-ray generator which will be built for XFEL.EU detector calibration purposes. In principle, the different features of X-ray spectra can be computed via the numerical solution of the electron-transport equation. However, this kind of solution is only possible for relatively simple interaction models and planar geometries. Therefore, Monte Carlo (MC) programs are the most suitable methods for the simulation of electron-induced X-ray spectra, mostly because they can incorporate realistic interaction cross sections and can be applied to targets with complex geometries. This is due to the fact that Monte Carlo methods permit to simulate the passage of radiation through matter taking into account all the relevant physical processes, and all particles (e.g. electrons and photons) can be tracked until they stop. To optimize the geometry of the generator as well as to estimate the intensity of the X-ray production, a simulation based on the general-purpose GEANT4 Monte Carlo tool-kit 23,24 was used. GEANT4 is a toolkit developed at CERN for the simulation of the passage of particles through matter. It is an object-oriented simulation framework which provides a diverse set of software components. All aspects of the simulation process, like the geometry of the system, the tracking of the particles through materials as well as the response of sensitive detector components, are included in the simulation. GEANT4 also provides a set of different models to describe the interaction of particles with matter across a wide energy range. The standard electromagnetic physics package implements electron, positron, photon and hadron interactions, but it is not optimized for low energy particles. Two specific low-energy electromagnetic models are available: the Livermore model, based on the Livermore cross-section and atomic data libraries, as well as the Penelope model, based upon the Penelope-code. ${ }^{25}$ For the present studies the Penelope model was used.

The geometry of the X-ray generator as implemented in the simulation is shown in Figure 1. Mono-energetic electrons with kinetic energies from $30 \mathrm{keV}$ to $100 \mathrm{keV}$ were hitting the target. The simulation was done for different target materials, incident angles $\alpha$, and electron beam diameters $\left(d_{\text {beam }}=0-1 \mathrm{~mm}\right)$. Each simulation consists of $4 \times 10^{6}$ electron. The procedure of the X-ray production consists in tracking a large number of incident electrons hitting the target, until they are absorbed or emitted from it, and calculating the amount of Bremsstrahlung and characteristic photons produced during the electrons' traverse of the target. The procedure starts with the definition of the electron source with the energy $E_{e}$. When the electrons hit the target, the code transports them inside the target material, producing secondary photons through Bremsstrahlung and ionization, until they are stopped.

The number of the X-ray quanta as a function of the incident angle $\alpha$ for the electrons with energy $E_{e}=100 \mathrm{keV}$ incident on a $5 \mathrm{~mm}$ thick $\mathrm{Cu}$ target is presented in Figure2. The maximum intensity of the X-ray beam is achieved for such geometries where the angle between the target and $x$-axis is smaller than $30^{\circ}$.

The X-ray spectra of different target materials for electron beam energy, $E_{e}=50$ and $100 \mathrm{keV}$, are shown in Figure 3. The results are normalized to the operation conditions corresponding to an electron beam current of 


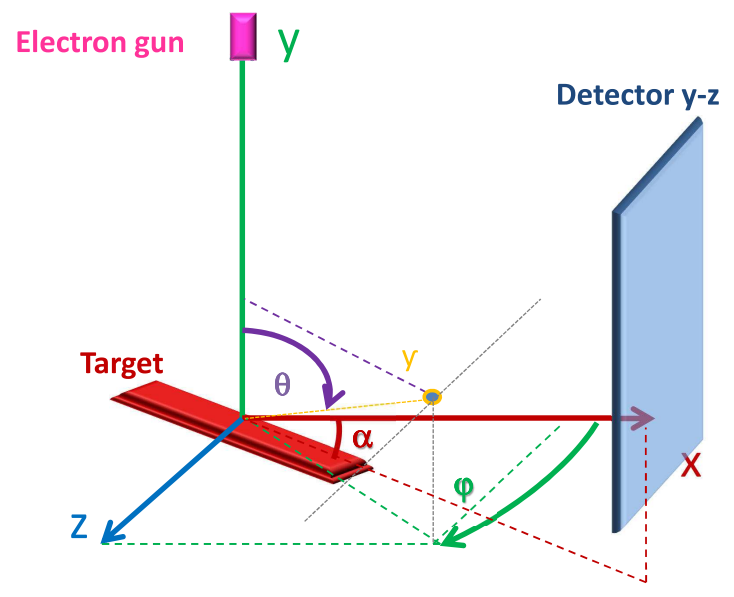

Figure 1. The geometry of the simulated setup.

$I_{e}=1 \mathrm{~mA}$, delivered in $\Delta t=1 \mathrm{~ns}$. The characteristic lines as well as the continuos spectra (Bremsstrahlung) are presented.

The number of X-ray photons produced in $\Delta t=1 \mathrm{~ns}$ as a function of the atomic number of the target material, $Z$, for the different electron energies $E_{e}=30,50$ and $100 \mathrm{keV}$ and $I_{e}=1 \mathrm{~mA}$ as well as efficiency of the $\mathrm{X}$-ray production are shown in Figure 4 The efficiency for X-ray production is varied from 0.4 up to $1.8 \%$ for electron energy $E_{e}=100 \mathrm{keV}$ and respectively lower for the lower energies.

The results presented in Figures 2 - 3 refer to the full angular range of the produced X-rays. Assuming the electron energy of $E_{e}=50 \mathrm{keV}$, the electron current of $I_{e}=20 \mathrm{~mA}$ and the pulse length of $50 \mathrm{~ns}$, the number of photons per pulse in the detector plane of size $20 \times 20 \mathrm{~cm}^{2}$ at $20 \mathrm{~cm}$ distance from the source is approx. $2 \times 10^{6}$. This yields two hits per pixel of size $200 \times 200 \mu \mathrm{m}^{2}$. The number of photons per pixel can be increased by using X-ray focussing optics (e.g. policapillary optics). Polycapillary focusing optics collect a large solid angle of X-rays from an X-ray source and focus them to a spot as small as a tens of $\mu \mathrm{m}$. The resulting X-ray flux density obtained is a few orders of magnitude higher than that obtained with a conventional pinhole collimator. A system design is under evaluation. Taking in to account the presented results, an electron source operated in pulsed mode with energies up to $50 \mathrm{keV}$ and electron current of $20 \mathrm{~mA}$ would be sufficient for the laboratory detector characterisation. The incident angle should not be larger than $30^{\circ}$ and the beam diameter shall range from 0.1 to $1 \mathrm{~mm}$.

\section{SOFTWARE DEVELOPMENT AT THE EUROPEAN XFEL}

The XFEL.EU is developing a dedicated software framework, Karabo, ${ }^{26}$ which integrates both controlling and data-analysis within a single design. Karabo is being written in both $\mathrm{C}++$ and Python. It is a distributed design, with messages being passed either via a broker or via peer-to-peer ( $\mathrm{p} 2 \mathrm{p}$ ) connections over a network. Its core are transparently distributable servers, which aggregate functionality via hot-plugable "devices". A device can be a control device, e.g. for steering a motor, a monitoring device, e.g. for measuring a pressure, or a computational device, e.g. for data processing. Interactivity is provided either from the command line interface (CLI) using IPython with automatic command-completion or from a PyQt-based graphical user interface (GUI) which aggregates (time-evolving) parameters, control and data-flows as well as state-, logging- and errorinformation. Within this GUI complex control and processing graphs can be laid out allowing for the design of e.g. data-analysis pipelines. 


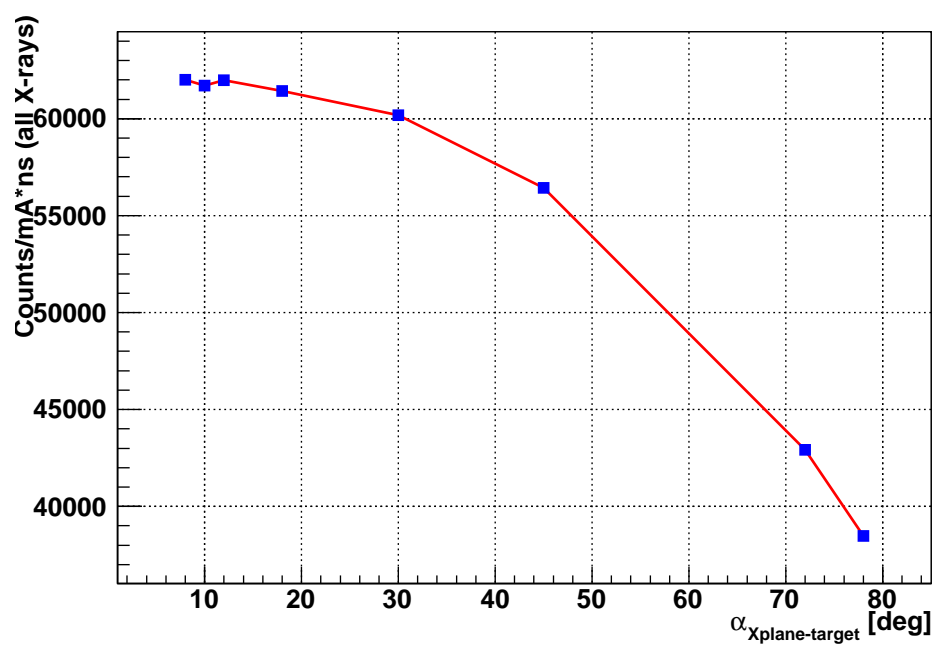

Figure 2. Number of X-ray quanta as a function of the incident angle $\alpha$ for the electrons with energy $E_{e}=100 \mathrm{keV}$ incident on $5 \mathrm{~mm}$ thick $\mathrm{Cu}$ target.
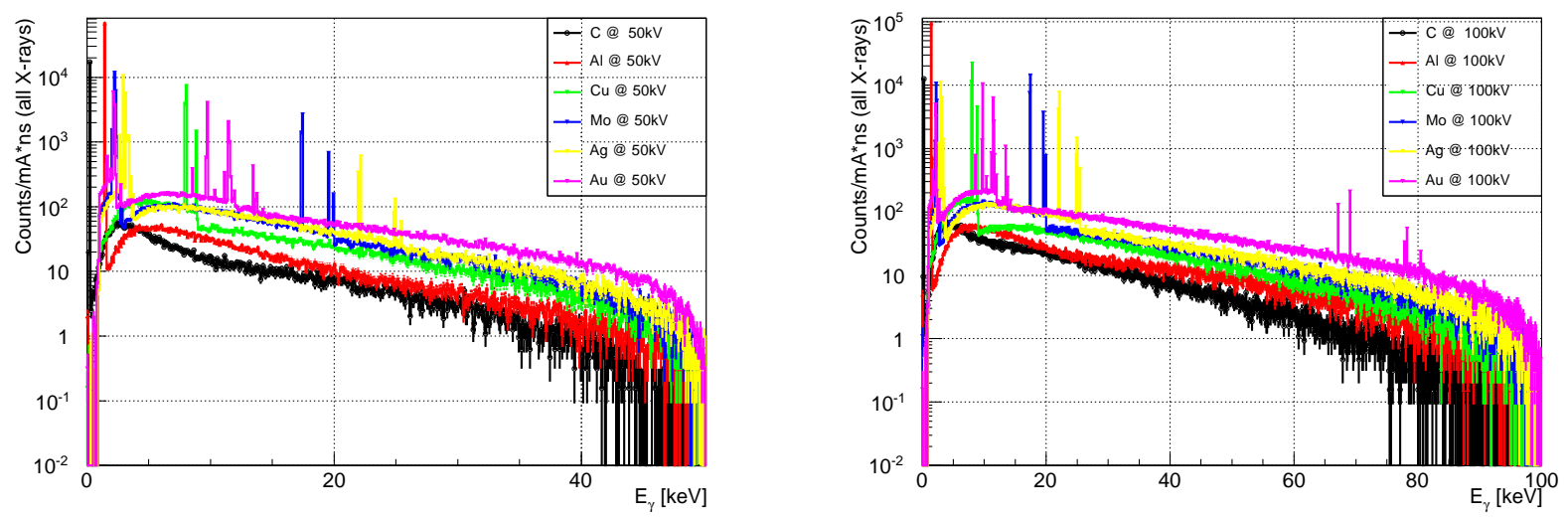

Figure 3. The X-ray spectra produced by the electrons with energy $E_{e}=50 \mathrm{keV}$ (left plot) and $100 \mathrm{keV}$ (right plot) hit on the different targets at the incident angle $\alpha=18^{\circ}$. 

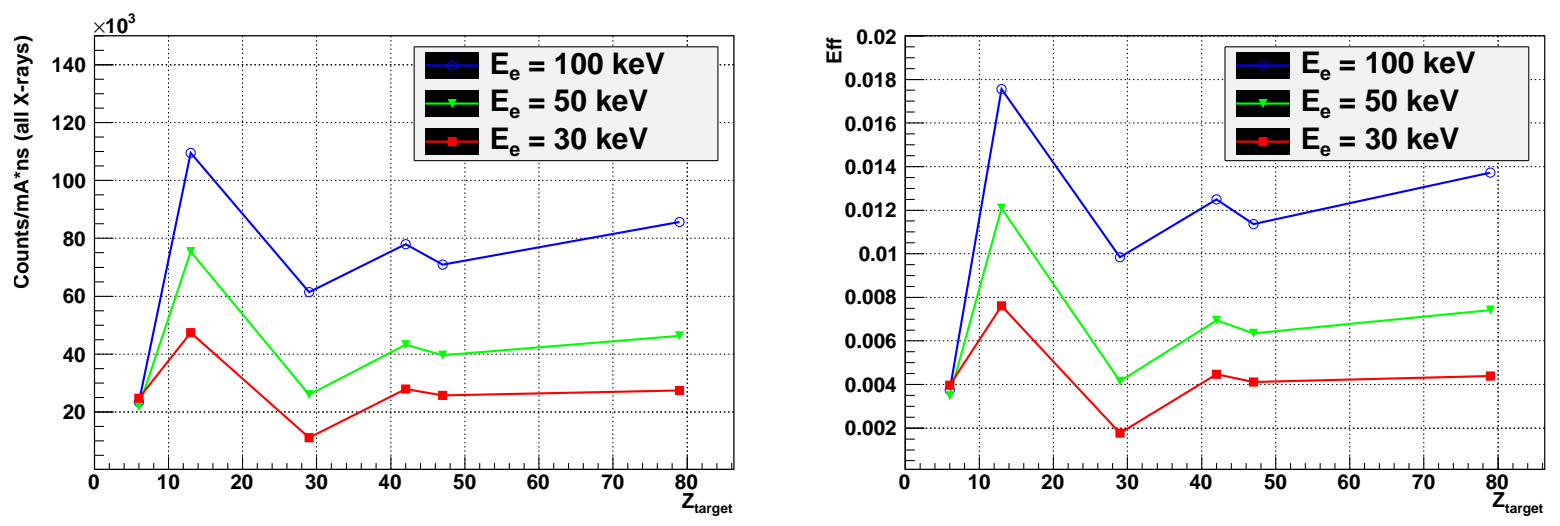

Figure 4. Number of X-ray quanta as a function of the atomic number of the target material (left plot) produced by the electrons with energies $E_{e}=30,50$ and $100 \mathrm{keV}$ hit on the target at the incident angle $\alpha=18^{\circ}$ and the efficiency of the $\mathrm{X}$-ray production (right plot).

\subsection{Detector-specific software in Karabo framework}

Within the Karabo framework there will be two flavors of detector specific software: control software and analysis/calibration software.

- Control software: includes software which is needed for controlling and monitoring the detector and closely related components. This includes e.g. sensor voltages, front-end electronic timings, cooling parameters, vacuum-pressures and corresponding safety-interlocks or mechanical positioning of the detector. Such software must necessarily be state-aware, i.e certain commands are only available in certain hardware contexts/modes.

- Calibration software: includes software which is used to apply calibration procedures to detector data resulting in data-sets which can be used for scientific analysis. Different calibration steps will generally be realized as individual software components (devices) which can be arranged into a processing pipeline. Calibration software also includes the analysis methods necessary for obtaining calibration parameters. Especially the application of calibration should be handled near-online, in order to provide scientists with a rapid feedback on their instrument and experiment.

\subsection{Simulation}

Computer-based simulations play an important role for an in-depth understanding of detector performance and characteristics such as the achievable dynamic range, the minimum noise or radiation induced performance deterioration. The required simulation tools are either inhouse developments, or derived from software used by the detector consortia during development.

In addition to estimating detector characteristics, Monte Carlo simulations are being used to optimize calibration hardware and procedures (e.g. X-ray tube simulation in Section 4.1.1). Furthermore, an end-to-end simulation is planned in the long term. This simulation aims to model as many aspects of the European XFEL as possible, starting from the electron source and ending with ready-to-analyze, simulated detector data of a simulated sample.

\section{CONCLUSION}

Detector development at the XFEL.EU for fast $(4.5 \mathrm{MHz})$ imaging detectors is supported by three external detector consortia. The first two-tile LPD system was delivered to the XFEL.EU for laboratory tests and characterization. The system will be tested on the PETRAIII beam at DESY in May. A prototype of the AGIPD detector is foreseen at the begining of 2014. In addition, as a backup solution and day-one-option two 
projects for low-repetition rate detectors are ongoing: pnCCD and FastCCD. Two additional detector activities have also started recently: 1D detector program for spectroscopy and diagnostics as well as small area $2 \mathrm{D}$ detectors.

In parallel to the detector development, the XFEL.EU detector group is building up the laboratory infrastructure needed for the detector calibration and characterization and develops the software for the data analysis and detector calibration and characterisation.

\section{ACKNOWLEDGMENTS}

We thank all the members of the detector consortia, DSSC, AGIPD and LPD for their effort in the XFEL.EU detector program and for the effective exchange of information. We thank the PSI detector group, Bernd Schmitt and Aldo Mozzanica in particular, and Andreas Ölsner, from Surface Concept, for the proficient communication.

\section{REFERENCES}

[1] Altarelli, M. et al., [European X-ray Free Electron Laser. Technical Design Report] (2006).

[2] Tschentscher, T., "Layout of the x-ray systems at the european XFEL," European XFEL GmbH, Technical Note 2011-001 (2011).

[3] Mancuso, A. P. et al., "Conceptual Design Report: Scientific Instrument Single Particles, Clusters and Biomolecues (SPB)," European XFEL GmbH, Technical Note 2011-007 (2011).

[4] Bressler, C. et al., "Technical Design Report: Scientific Instrument FXE," European XFEL GmbH, Technical Note 2012-008 (2012).

[5] Madsen, A. et al., "Conceptual Design Report: Scientific Instrument MID ," European XFEL GmbH, Technical Note 2011-008 (2011).

[6] Tschentscher, T. et al., "Conceptual Design Report: Scientific Instrument HED ," to be published .

[7] Meyer, M. et al., "Technical Design Report: Scientific Instrument SQS," European XFEL GmbH, Technical Note 2012-007 (2012).

[8] Scherz, A. et al., "Conceptual Design Report: Scientific Instrument SCS ," to be published.

[9] Hart, M. et al., "Development of the LPD, a High Dynamic Range Pixel Detector for the European XFEL," IEEE proceedings (to be published) (2012).

[10] Henrich, B. et al., "The adaptive gain integrating pixel detector AGIPD a detector for the European XFEL," Nucl. Instrum. Methods. 633, Supplement 1(0), S11 - S14 (2011). 11th International Workshop on Radiation Imaging Detectors (IWORID).

[11] Shi, X. et al., "Challenges in chip design for the AGIPD detector," Nucl. Instrum. Methods. 624(2), 387 391 (2010).

[12] Schwandt, J. et al., "Design of the AGIPD Sensor for the European XFEL," JINST 8, C01015 (2013).

[13] Schwandt, J. et al., "Optimization of the Radiation Hardness of Silicon Pixel Sensors for High X-ray Doses using TCAD Simulations," JINST 7, C01006 (2012).

[14] Becker, J. et al., "Performance tests of an AGIPD 0.4 assembly at the beamline P10 of PETRA III," (2013). arXiv:1303.2505 [physics.ins-det].

[15] Porro, M. et al., "Development of the DEPFET sensor with signal compression: A large format x-ray imager with mega-frame readout capability for the european XFEL," IEEE Trans. Nucl. Sci. PP(99), 1 (2012).

[16] Seibert, M. M. et al., "Single mimivirus particles intercepted and imaged with an X-ray laser," Nature 470, 78-81 (Feb. 2011).

[17] Huang, X. et al., "Signal-to-noise and radiation exposure considerations in conventional and diffraction x-ray microscopy," Optics Express 17(16), 13541 (2009).

[18] Giewekemeyer, K., Turcato, M., and Mancuso, A., "Detector geometries for coherent x-ray diffractive iamging at the SPB beamline," European XFEL GmbH, Technical note, to be published.

[19] \{Giewekemeyer, Klaus\}, [Personal Communication] (2013).

[20] Boutet, S. et al., "High-resolution protein structure determination by serial femtosecond crystallography," Science (2012). 
[21] Denes, P. et al., "A fast, direct x-ray detection charge-coupled device," Rev. Sci. Instrum. 80 (2009).

[22] Strüder, L. et al., "Large-format, high-speed, X-ray pnCCDs combined with electron and ion imaging spectrometers in a multipurpose chamber for experiments at 4th generation light sources," Nucl. Instrum. Methods. 614, 483-496 (Mar. 2010).

[23] Agostinelli, S. et al., "Geant4 - a simulation toolkit," Nucl. Instrum. Methods. 506(3), 250 - 303 (2003).

[24] Allison, J. et al., "Geant4 developments and applications," IEEE Trans. Nucl. Sci. 53, 270-278 (2006).

[25] Salvat, F. et al., [PENELOPE - A code system for Monte Carlo Simulation of Electron and Photon Transport], Nuclear Energy Agency, OECD (2011). http://www.oecd-nea.org/dbprog/penelope.pdf.

[26] \{Heisen, Burkhard\}, [Personal Communication] (2013). 


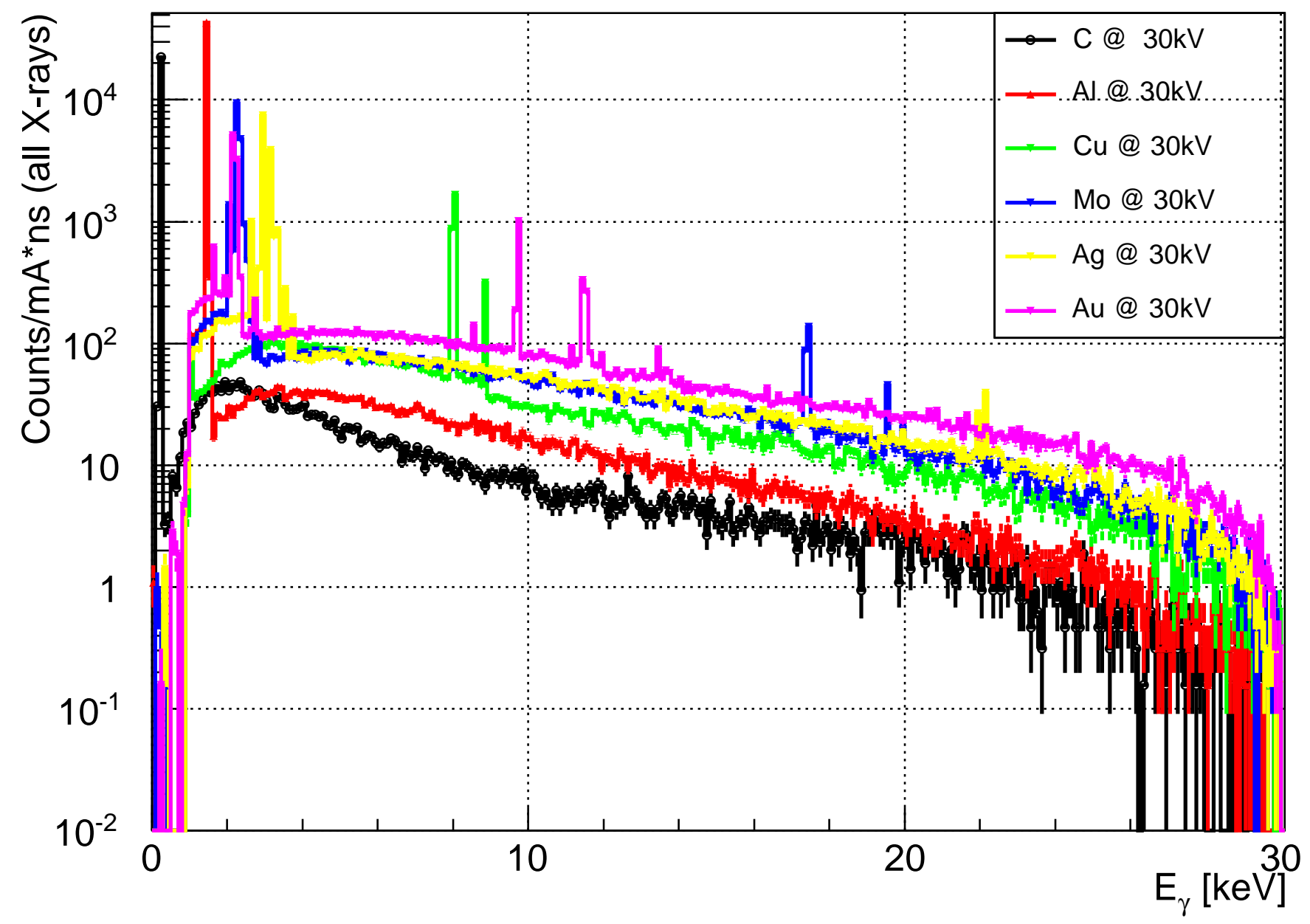

